\title{
Article \\ Modeling of Metabolic Equivalents (METs) during Moderate Resistance Training Exercises
}

\author{
Muhammad Adeel ${ }^{1,2}$, Chien-Hung Lai ${ }^{3,4}$, Chun-Wei Wu ${ }^{2}$, Jiunn-Horng Kang ${ }^{3,4}$, Jian-Chiun Liou ${ }^{2}$, \\ Hung-Chou Chen ${ }^{3,5}{ }^{(-)}$, Bor-Shing Lin ${ }^{6}{ }^{-}$, Meng-Jyun Hong ${ }^{2}$, Chun-Ta Feng ${ }^{2}$ and Chih-Wei Peng $1,2,7, *(1)$
}

check for updates

Citation: Adeel, M.; Lai, C.-H.; Wu, C.-W.; Kang, J.-H.; Liou, J.-C.; Chen, H.-C.; Lin, B.-S.; Hong, M.-J.; Feng, C.-T.; Peng, C.-W. Modeling of Metabolic Equivalents (METs) during Moderate Resistance Training Exercises. Appl. Sci. 2021, 11, 8773. https://doi.org/10.3390/ app11188773

Academic Editor: Jesús García Pallarés

Received: 21 July 2021

Accepted: 17 September 2021

Published: 21 September 2021

Publisher's Note: MDPI stays neutral with regard to jurisdictional claims in published maps and institutional affiliations.

Copyright: (c) 2021 by the authors Licensee MDPI, Basel, Switzerland. This article is an open access article distributed under the terms and conditions of the Creative Commons Attribution (CC BY) license (https:// creativecommons.org/licenses/by/ $4.0 /)$.
1 International Ph.D. Program in Biomedical Engineering, College of Biomedical Engineering, Taipei Medical University, Taipei 110, Taiwan; d845108004@tmu.edu.tw

2 School of Biomedical Engineering, College of Biomedical Engineering, Taipei Medical University, Taipei 110, Taiwan; george.jasonbiolab@gmail.com (C.-W.W.); jcliou@tmu.edu.tw (J.-C.L.); b812107003@tmu.edu.tw (M.-J.H.); dar197908@tmu.edu.tw (C.-T.F.)

3 Department of Physical Medicine and Rehabilitation, School of Medicine, College of Medicine, Taipei Medical University, Taipei 110, Taiwan; chlai@tmu.edu.tw (C.-H.L.); jhk@tmu.edu.tw (J.-H.K.); 10462@s.tmu.edu.tw (H.-C.C.)

4 Department of Physical Medicine and Rehabilitation, Taipei Medical University Hospital, Taipei 110, Taiwan

5 Department of Physical Medicine and Rehabilitation, Shuang Ho Hospital, Taipei Medical University, New Taipei 235, Taiwan

6 Department of Computer Science and Information Engineering, National Taipei University, New Taipei 237, Taiwan; bslin@mail.ntpu.edu.tw

7 School of Gerontology Health Management, College of Nursing, Taipei Medical University, Taipei 110, Taiwan * Correspondence: cwpeng@tmu.edu.tw; Tel./Fax: +886-2-2736-1661 (ext. 3070)

Abstract: Energy expenditure through metabolic equivalent (MET) prediction during resistance exercises in humans can be modeled by using cardiorespiratory parameters. In this study, we aimed to predict MET during six moderate-intensity resistance training sessions consisting of three different exercises. Eleven participants were recruited into two groups; an untrained ( $\mathrm{n}=5$; with no resistance training experience) and a trained group $(n=6$; with 2 months resistance training experience). Each participant completed six training sessions separated with a rest interval of 1-2 days. While wearing a mask for indirect calorimetric measurements using Cortex Metalyzer 3B, each participant performed training sessions consisting of three types of dumbbell exercises: shoulder press, deadlift, and squat. The metabolic equivalents (METs), respiratory exchange ratio (RER), heart rate (HR), systolic blood pressure (SBP), diastolic blood pressure (DBP), blood lactate (BL), and Borg rate of perceived exertion (RPE) were measured. The MET was predicted using generalized estimating equations (GEE) for repeated measure data collected during exercise and rest periods. It was observed that during exercise period, RER, HR, SBP, and BL for the training group (QIC $=187,95 \% \mathrm{CI}=-0.012 \sim 0.915, p=0.000^{*} \sim 0.033^{*}$ ) while RER, HR, SBP, DBP, and RPE $\left(\mathrm{QIC}=48,95 \% \mathrm{CI}=-0.024 \sim 0.422, p=0.000^{*} \sim 0.002^{*}\right)$ during resting period for untrained group significantly predicted MET for moderate-intensity interval resistance training. It is concluded that the cardiorespiratory variables are significantly related to MET. During exercise, RER and HR significantly predicted MET for both groups along with additional parameters of SBP and BL for the training group. While during the resting period, RER, HR, SBP, DBP, and RPE related significantly for untrained and BL for training group respectively.

Keywords: strength training; METs; GEE modeling; cardiorespiratory variables

\section{Introduction}

For decades, resistance training (RT) has been one of the most popular modes of exercise used by athletes and exercise populations [1]. According to the American College of Sports Medicine (ACSM), American Heart Association (AHA), and the Surgeon General's Report on Physical Activity and Health, strength training is important for better health 
and well-being. It improves strength, anaerobic capacity, body composition, bone density, flexibility, and physical function [1]. The recommended volume of resistance exercise as suggested by ACSM should include at least one set of 8-12 repetitions of each one of 8-10 exercises involving the major muscle groups to gain health and fitness benefits [2].

Given the rise in RT participation and the ongoing increase in the number of overweight people [1], a precise measurement of RT's energy expenditure would be beneficial. But there are several problems in measuring energy expenditure during resistance exercises including involvement of large mass (which significantly cause higher energy expenditure) [3], number of sets, rest period, number of repetitions, speed of movement, and load [4]. The greater number of variables makes it difficult to compare results with previous studies. In addition, individual factors like age, gender, body composition, and fitness level are also taken into account as possible intervening variables [4]. However, because RT causes metabolic demands in all three energy systems, indirect calorimetry cannot precisely quantify the energy utilization of moderate-to-high intensity RT [1]. Despite this, Wilmore et al. [5] were the first to calculate the metabolic cost of RT during exercise and recovery using indirect calorimetry. With underlying limitations, indirect calorimetry is the standard clinical approach to assess the energy expenditure of healthy or diseased individuals over time [6] during physical activity.

The Compendium of Physical Activities reported the metabolic equivalent (MET) as the ratio of work metabolic rate to a standard resting metabolic rate of $1.0(4.184 \mathrm{~kJ}) / \mathrm{kg} / \mathrm{h}$, $1 \mathrm{MET}$ is considered a resting metabolic rate attained during quiet sitting. The Compendium lists activities as multiples of the resting MET level, ranging from 0.9 (sleeping) to $18 \mathrm{METs}$ (running at $10.9 \mathrm{mph}$ ) [7] and resistance training refers to 3.5-5 METs [8].

There are many research studies conducted with variable resistance exercise parameters to derive metabolic equations. A study by Gustavo et al. 2020 reported a regression equation for the energy cost of a single resistance training session consisting of bench press, pec deck, squat, lat pulldown, biceps curl, triceps extension, hamstring curl, and crunch in healthy males at $75 \%$ repetition maximum $(\mathrm{RM}): \mathrm{Y}=-473.595+-1.211\left(\mathrm{X}_{1}\right)+17.572\left(\mathrm{X}_{2}\right)$ $\left(\mathrm{R}^{2}=0.61, p<0.05\right)$. In which $\mathrm{X}_{1}=$ load-volume (no. of sets $\mathrm{x}$ no. of repetitions) and $\mathrm{X}_{2}=$ session time (minutes) [9]. Another study predicted resting energy expenditure (REE) equations in master athletes using body weight, gender, age, height, fat-free mass (FFM), fat mass (FM), temperature, training hours, phase angle, and kind of sports $\left(\mathrm{R}^{2}=0.55-0.69\right)$. They concluded that body composition and ambient temperature are the primary variables for determining REE [10].

Up to our knowledge, no research study is found to relate MET with cardiorespiratory variables of moderate resistance exercises at $60 \%$ of $1 \mathrm{RM}$. Because it is important to determine which cardiorespiratory variable has a strong relationship in predicting MET and compare these variables for the untrained and trained participants during moderate resistance exercises. This study aimed to model METs with various cardiorespiratory variables like respiratory exchange ratio (RER), heart rate (HR), blood pressure (systolic and diastolic-SBP and DBP), blood lactate (BL), and Borg rate of perceived exertion (RPE) scale during three dumbbell resistance training exercises (shoulder press, deadlift, and squat). The primary goal of the study was to determine the multi-linear relation among MET as a dependent while RER, HR, SBP, DBP, BL, and RPE as independent variables using generalized estimating equations (GEE). The secondary aim was to compare untrained with trained group participants' cardiorespiratory variables.

\section{Materials and Methods}

Participant Recruitment: A single-blinded controlled study was conducted at Taipei Medical University Hospital, and the protocol was approved by the TMU-Joint Institutional Review Board (IRB no.: N202004023). The trial was registered at ClinicalTrials.gov (accessed on 11 September 2021) (NCT04532905). A total of 12 young male and female participants were recruited into two groups between December 2020 and May 2021 through convenience sampling. Among these, one was excluded, and the untrained group contained five 
participants (with no resistance training experience) and trained group six participants (with 2 months of resistance training experience). Each participant signed a written consent form before the commencement of the study. The participants were informed about the objectives and potential risks of the research.

The inclusion criteria were: (1) a healthy male or female individual between $20 \sim 40$ years of age; (2) no metabolic, systematic, or musculoskeletal disease or injury from the last 6 months; (3) no recent surgical procedure which could limit exercise training; (4) taking no medications, especially sedatives, antidepressants, antihypertensive, etc.; and (5) physically fit according to the Physical Activity Readiness Questionnaire (PAR-Q) [11] (Figure 1). Functional outcome measurements and data analyses were performed by trained research staff who were not involved in the intervention. Participants were blinded for the exercise order in this study.

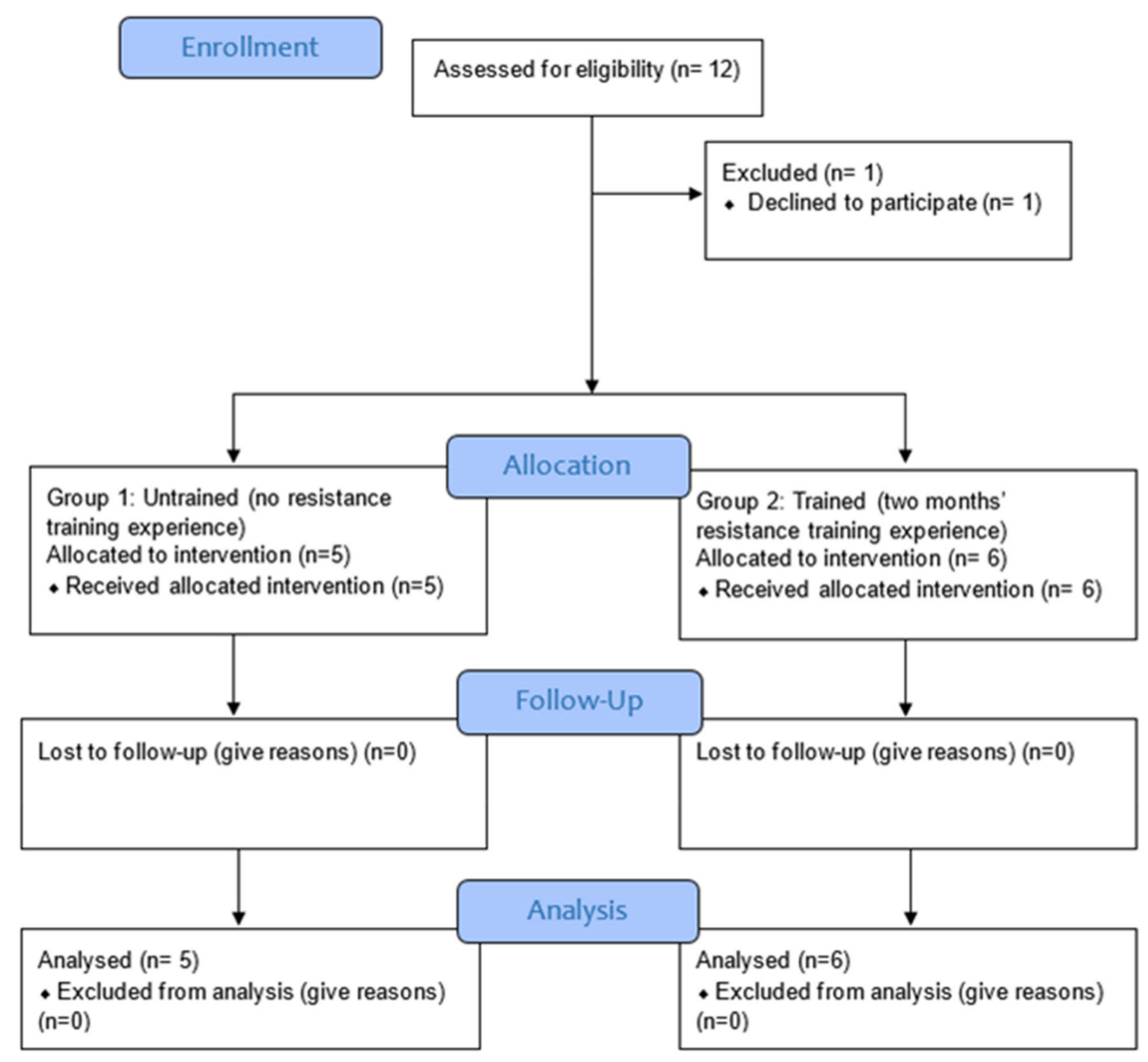

Figure 1. Study flow diagram.

Experimental procedure: Each participant visited the training room for eight separate sessions where testing and data collection were completed. All participants were instructed to eat a meal 2 4 $\mathrm{h}$ before testing, to avoid alcohol and caffeine ingestion for $24 \mathrm{~h}$ before testing, and to refrain from strenuous exercise for $24 \sim 48 \mathrm{~h}$ before testing [12].

Session 1: During the first visit, baseline body weight $(\mathrm{kg})$, height $(\mathrm{cm})$, body-mass index (BMI), and body fat percentage were measured using a Karada scan-371 body scale (Omron, Kyoto, Japan). Each participant was evaluated by the PAR-Q for physical fitness; 
the testing and training procedure was explained to them by an expert researcher. In this session, participants performed an incremental cycling test using Cortex Metalyzer 3B (Cortex, Leipzig, Germany) while wearing a mask to assess cardiopulmonary fitness and familiarity with using the mask during physical activity. The flow and gas sensors were calibrated before every test. The temperature and humidity of the room were respectively set to $22-27^{\circ} \mathrm{C}$ and $52 \% \sim 64 \%$. The incremental bicycle testing protocol was conducted when the participant pedaled the bicycle at approximately 60 revolutions per minute (rpm) against 25 watts of resistance. The resistance was increased by 25 watts at the beginning of each two-minute stage [13]. The test was stopped when two of three occurred; a respiratory exchange ratio (RER) $\geq 1.1$, an HR within 10 beats or over their theoretical aged-predicted maximal HR (220-age), and an expression of RPE $\geq 16 / 20$ [14].

Session 2: During this session, each participant performed a maximum of three to five sets to achieve their maximum $1 \mathrm{RM}$ for dumbbell shoulder press, deadlift, and squat exercises at a cadence of $1.5 \mathrm{~s}$ up and $1.5 \mathrm{~s}$ down using an audible metronome. An audible cadence was used to control for potential variations in the lifting cadence of the participants. Briefly, participants performed a warm-up consisting of eight to ten repetitions using a lightweight, three to five repetitions using a moderate weight, and one to three repetitions using a heavyweight. After the warm-up sets, the $1 \mathrm{RM}$ strength was tested by increasing the resistance on subsequent attempts until the participants were unable to complete an attempt using a proper technique through a full range of motion [15]. Between each set, the participant was given a 2 4 min rest interval. Between the $1 \mathrm{RM}$ shoulder press, deadlift, and squat there was a 10 15 min rest period when participants were allowed to walk, perform light dynamic movements, and consume small amounts of water [16]. After determining $60 \%$ of $1 \mathrm{RM}$, participants performed twothree familiarization sessions on separate days before starting the formal training sessions.

Sessions 3 8: Before the start of the training session, every participant did prestretching and warm up for 5-10 min. Participants performed six RT sessions on separate days in a randomized order (Table 1), each at the same time of day, which consisted of the shoulder press, deadlift, and squat exercises at $60 \%$ of $1 \mathrm{RM}$, three sets of 10 repetitions at a cadence of $1.5 \mathrm{~s}$ up and $1.5 \mathrm{~s}$ down using an audible metronome to control for potential variations in the lifting cadence of participants. Between each set, there was 2 min rest period and $8 \mathrm{~min}$ rest interval between each type of exercise. A minimum of $24 \sim 48 \mathrm{~h}$ of a rest interval between each resistance training session was included.

Table 1. Order of exercise sequences (interval resistance training).

\begin{tabular}{ccccc}
\hline \multirow{2}{*}{ Exercise Order } & Training & \multicolumn{3}{c}{ Exercises } \\
\cline { 3 - 5 } & Sessions & $\mathbf{1}$ & $\mathbf{2}$ & $\mathbf{3}$ \\
\hline \multirow{2}{*}{ Sequence 1 } & Session 1 & Shoulder press & Deadlift & Squat \\
\cline { 2 - 5 } & Session 2 & Shoulder press & Deadlift & Squat \\
\hline \multirow{2}{*}{ Sequence 2 } & Session 3 & Deadlift & Shoulder press & Squat \\
\cline { 2 - 5 } & Session 4 & Deadlift & Shoulder press & Squat \\
\hline \multirow{2}{*}{ Sequence 3 } & Session 5 & Squat & Shoulder press & Deadlift \\
\cline { 2 - 5 } & Session 6 & Squat & Shoulder press & Deadlift \\
\hline
\end{tabular}

Each exercise was performed with dumbbells on a separate day total consisting of six training sessions.

The oxygen consumption $\left(\mathrm{VO}_{2}\right)$ was recorded during each training session through a breath-by-breath analysis using Cortex Metalyzer 3B while wearing a mask. Before beginning training, participants were provided with an explanation of the Borg rate of perceived exertion scale (6-20; RPE). The $\mathrm{VO}_{2}, \mathrm{RER}$, and $\mathrm{HR}$ were recorded for a total of 52:30 min for each training session, including the resting (10 $\mathrm{min})$, exercise $(30 \mathrm{~s} \times$ nine sets), rest after exercise (2-8 $\mathrm{min})$, and recovery $(10 \mathrm{~min}$ ) periods. Blood pressure (systolic and diastolic-SBP and DBP), RPE, and blood lactate (BL) were recorded before the start of 
training and immediately after each exercise using a portable Omron sphygmomanometer (Omron Healthcare, Inc., IL, USA), RPE scale, and portable THE EDGE Blood Lactate Monitoring System (ApexBio, Hsinchu, Taiwan). For blood lactate monitoring, blood samples were collected and asepsis was performed with $70 \%$ ethyl alcohol on the distal fingertips of the left or right hand. The puncture was performed using disposable lancets, and a suspended drop of blood was applied to a lactate test strip for analysis on a lactate meter [12] (Figure 2).

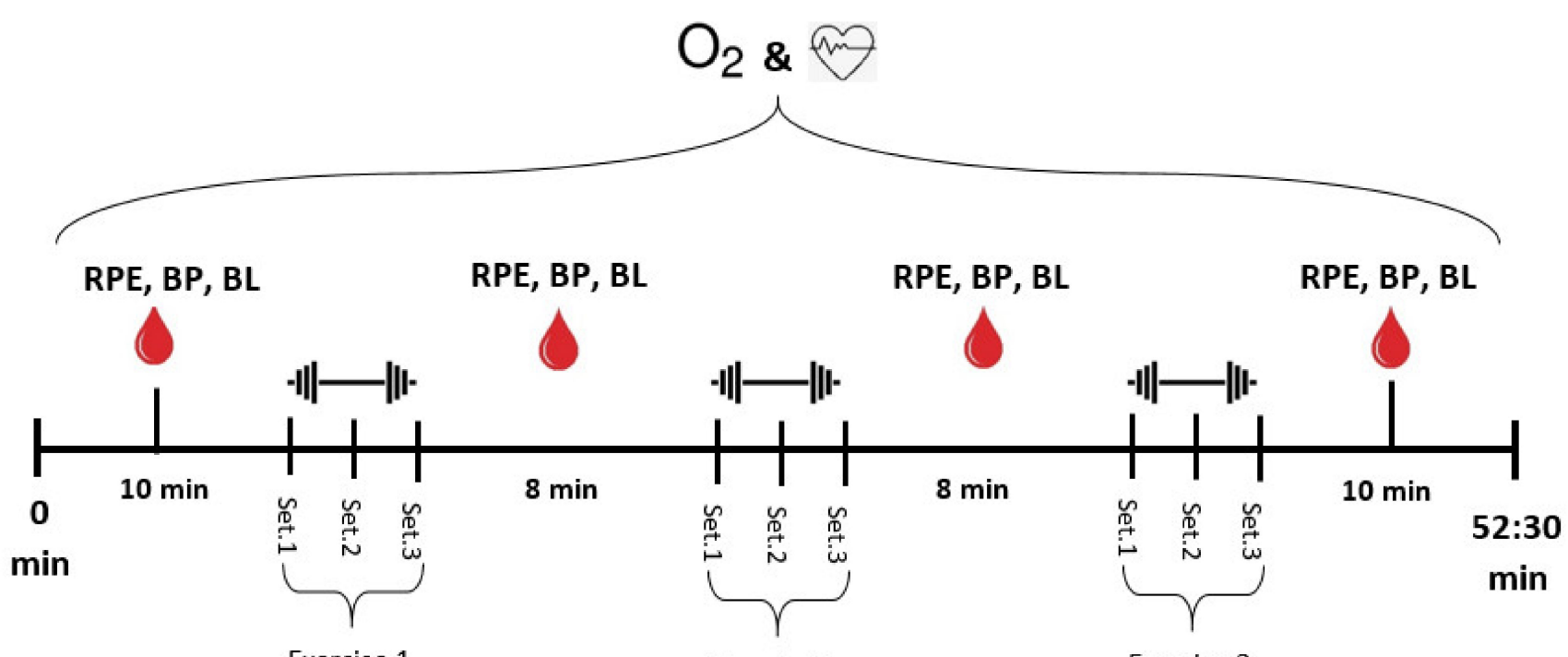

Exercise.1

Exercise.2

Exercise.3

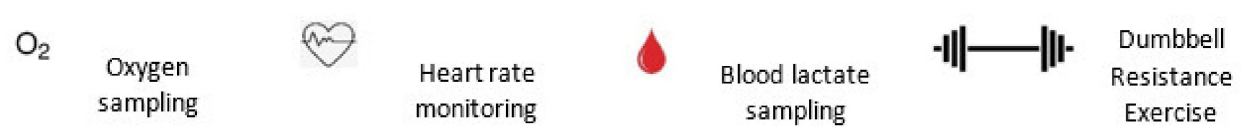

Figure 2. Experiment training protocol. RPE, Borg rate of perceived exertion; systolic and diastolic blood pressure (SBP and DBP); and BL, blood lactate; these were measured a total of four times; before start of training, after exercise 1, exercise 2, and exercise 3. Each exercise set took $30 \mathrm{~s}$ and 2 min rest interval between each set and 8 min rest interval between each type of exercise.

Outcome measures: The recorded variables were $\mathrm{VO}_{2}(\mathrm{ml} / \mathrm{kg} / \mathrm{min}), \mathrm{VO}_{2}(\mathrm{~L} / \mathrm{min})$, METs, RER, HR (beats per min (bpm)), SBP (mmHg), DBP (mmHg), BL (mg/dl), and RPE score. For each exercise of six sessions, the METs, RER, and HR during exercise, rest, and recovery periods while blood pressures, RPE, and BL recorded during rest are presented as average values.

Statistical analysis: Raw data from the Cortex metalyzer were exported and processed in Excel software. For statistical analysis, SPSS software (IBM SPSS Statistics vs. 19, Armonk, NY, USA) was used. The normality of the data was evaluated by a bell-shaped histogram. Study demographics are presented in the form of descriptive statistics (Table 2). Our study data were continuous repeated measures, so for modeling MET with multiple repeated variables, generalized estimating equations (GEE) [17] with backward deletion method were used in SPSS. Variables for the GEE analysis included METs, RER, and HR during exercise and rest periods, and SBP, DBP, BL, and RPE during the resting period were entered. Two categories of GEE models were computed: 
Table 2. Study baseline characteristics $(n=11)$.

\begin{tabular}{ccc}
\hline Groups & Untrained $(\mathbf{n}=\mathbf{5 )}$ & Trained (n = 6) \\
\hline Age (years) & $22.00 \pm 2.00$ & $25.83 \pm 3.66$ \\
\hline Male $/$ female & $0 / 5$ & $4 / 2$ \\
\hline Weight $(\mathrm{kg})$ & $53.32 \pm 3.38$ & $81.68 \pm 19.48$ \\
\hline Height $(\mathrm{cm})$ & $158.60 \pm 5.03$ & $173.33 \pm 10.38$ \\
\hline Body mass index $\left(\mathrm{kg} / \mathrm{m}^{2}\right)$ & $21.26 \pm 1.66$ & $26.87 \pm 4.54$ \\
\hline \% Body fat & $23.60 \pm 2.43$ & $26.22 \pm 1.86$ \\
\hline Habitual activity $/$ week $(\mathrm{h})$ & $3.14 \pm 0.92$ & $4.40 \pm 1.13$ \\
\hline RM 60\% of shoulder press & $16.08 \pm 3.68$ \\
\hline RM $60 \%$ of deadlift $(\mathrm{kg})$ & $9.10 \pm 1.60$ & $34.42 \pm 3.32$ \\
\hline RM 60 \% of squat $(\mathrm{kg})$ & $19.50 \pm 4.11$ & $32.33 \pm 3.76$ \\
\hline mean \pm SD: RM: repetition maximum: Training loads in kilogram $(\mathrm{kg})$ for both side dumbbells.
\end{tabular}

(1) Exercise models: During exercise, group models were predicted using the data of exercise period including MET, RER, HR, and resting period including SBP, DBP, BL, and RPE for analysis. The factor considered was exercise type (consisted of three exercises: shoulder press, deadlift, and squat), dependent variable MET, and covariates or independent variables were RER, HR, SBP, DBP, BL, and RPE for untrained and trained group models.

(2) Resting models: During resting periods, group models were also computed using the same variables as explained above but the resting data for MET, RER, HR, SBP, DBP, $\mathrm{BL}$, and RPE were used. The estimate (ß), standard error (SE), 95\% confidence interval (CI), and $p$-value were considered for GEE modeling [18]. A narrower $95 \% \mathrm{CI}$ indicates a more accurate estimate, while a wider $95 \% \mathrm{CI}$ indicates a less accurate estimate [19]. The fit of GEE models was compared between exercise and resting periods using quasi-likelihood under independence model criterion (QIC) which means the smaller the QIC has the better the model fit [20]. For all six training sessions: METs, RER, HR, SBP, DBP, BL, and RPE were compared with the mean \pm standard deviation (SD) and $p$-values using one-way ANOVA between two groups. The significance level was set to $p<0.050$.

\section{Results}

\subsection{Baseline Characteristics of Participants}

Twelve participants were recruited in this study, out of which one was excluded. Based on their resistance training experience, a total of eleven participants were randomly allocated into untrained $(n=5)$ and trained $(n=6)$ groups. Baseline features of all participants including age (years), gender (male/female), body weight $(\mathrm{kg})$, height $(\mathrm{cm})$, BMI $\left(\mathrm{kg} / \mathrm{m}^{2}\right)$, percentage body fat, habitual activity/week (h), and $60 \%$ repetition maximum (RM) training load of shoulder press, deadlift, and squat are presented in Table 2. No participant in either group experienced any adverse events during or after the six training sessions.

\subsection{Modeling of MET during Six Training Sessions (Group Vise during Exercise Period)}

During the exercise period of six training sessions, the GEE model significantly predicted MET. For the untrained group, the respiratory exchange ratio (RER) $\left(p=0.004^{*}\right.$ and $95 \% \mathrm{CI}=0.152 \sim 0.789)$ and heart rate $(\mathrm{HR})\left(p=0.000^{*}\right.$ and $\left.95 \% \mathrm{CI}=0.009 \sim 0.022\right)$ were significantly predicted MET while all other parameters including systolic blood pressure (SBP), diastolic blood pressure (DBP), blood lactate (BL), and Borg rate of perceived exertion (RPE) did not show a significant relationship with MET. For the training group, RER $\left(p=0.021^{*}\right.$ and $\left.95 \% \mathrm{CI}=0.073 \sim 0.915\right), \mathrm{HR}\left(p=0.001^{*}\right.$ and $\left.95 \% \mathrm{CI}=0.005 \sim 0.018\right)$, SBP 
( $p=0.033^{*}$ and $\left.95 \% \mathrm{CI}=-0.011 \sim 0.000\right)$, and BL $\left(p=0.000^{*}\right.$ and 95\% $\left.\mathrm{CI}=-0.012 \sim-0.004\right)$ reached a significant level in modeling MET. The QIC for exercise models were 74 and 187 while for resting models were 48 and 95 for untrained and trained groups, respectively (Tables 3 and 4).

Table 3. Generalized estimating equations for metabolic equivalents (METs) prediction during six training sessions (exercise period). $(n=11)$.

\begin{tabular}{ccccccc}
\hline Groups & Model & Parameter & Estimate (B) & SE & $\begin{array}{c}\text { 95\% CI } \\
\text { (Lower Upper) }\end{array}$ & $p$-Value \\
\hline \multirow{2}{*}{ UTr } & Model 1 & Intercept & 0.443 & 0.527 & $-0.590 \sim 1.475$ & 0.401 \\
$(\mathrm{n}=5)$ & RER & 0.471 & 0.162 & $0.152 \sim 0.789$ & 0.004 * \\
& & HR & 0.016 & 0.004 & $0.009 \sim 0.022$ & $0.000 *$ \\
\hline & & Intercept & 2.894 & 0.920 & $1.092 \sim 4.697$ & 0.002 \\
Tr & Model 2 & RER & 0.494 & 0.215 & $0.073 \sim 0.915$ & $0.021 *$ \\
$(\mathrm{n}=6)$ & HR & 0.012 & 0.003 & $0.005 \sim 0.018$ & $0.001 *$ \\
& & SBP & -0.006 & 0.003 & $-0.011 \sim 0.000$ & $0.033 *$ \\
& & BL & -0.008 & 0.002 & $-0.012 \sim-0.004$ & $0.000 *$ \\
\hline
\end{tabular}

UTr, untrained; Tr, trained groups; * indicates a significant difference $p<0.050$. SE, standard error; $95 \% \mathrm{CI}$ confidence intervals; QIC, quasi-likelihood under independence model criterion, RER, respiratory exchange ratio; $\mathrm{HR}$, heart rate; SBP, systolic blood pressure; BL, blood lactate.

Table 4. Generalized estimating equations for metabolic equivalents (METs) prediction during six training sessions (resting period). $(\mathrm{n}=11)$.

\begin{tabular}{|c|c|c|c|c|c|c|}
\hline Groups & Model & Parameter & Estimate (ß) & SE & $\begin{array}{c}95 \% \text { CI } \\
\text { (Lower Upper) }\end{array}$ & $p$-Value \\
\hline \multirow{6}{*}{$\begin{array}{c}\mathrm{UTr} \\
(\mathrm{n}=5)\end{array}$} & \multirow{6}{*}{$\begin{array}{l}\text { Model } 1 \\
\text { (QIC 48) }\end{array}$} & Intercept & 1.971 & 0.232 & $1.517 \sim 2.426$ & 0.000 \\
\hline & & RER & 0.329 & 0.047 & $0.237 \sim 0.422$ & $0.000 *$ \\
\hline & & HR & 0.006 & 0.002 & $0.003 \sim 0.009$ & $0.000 *$ \\
\hline & & SBP & 0.008 & 0.001 & $0.007 \sim 0.010$ & $0.000 *$ \\
\hline & & DBP & -0.021 & 0.002 & $-0.024 \sim-0.017$ & $0.000 *$ \\
\hline & & RPE & 0.034 & 0.011 & $0.013 \sim 0.056$ & $0.002 *$ \\
\hline \multirow{2}{*}{$\begin{array}{c}\operatorname{Tr} \\
(n=6)\end{array}$} & \multirow{2}{*}{$\begin{array}{l}\text { Model } 2 \\
\text { (QIC 95) }\end{array}$} & Intercept & 3.610 & 0.116 & $3.383 \sim 3.838$ & 0.000 \\
\hline & & $\mathrm{BL}$ & -0.001 & 0.001 & $-0.003 \sim 0.000$ & $0.045 *$ \\
\hline
\end{tabular}

UTr, untrained; Tr, trained groups; * indicates a significant difference $p<0.050$. SE, standard error; 95\% CI confidence intervals; QIC, quasi-likelihood under independence model criterion, RER, respiratory exchange ratio $\mathrm{HR}$, heart rate; SBP, systolic blood pressure; DBP, diastolic blood pressure; BL, blood lactate; RPE, Borg rate of perceived exertion.

\subsection{Modeling of MET during Six Training Sessions (Group Vise during the Rest Period)}

During the rest period, after each set and exercise, GEE models were also calculated similarly to the method used for the exercise period. As shown in Table 4, RER, HR, SBP, DBP, and RPE were significantly related with MET ( $p=0.000 \sim 0.002 *$ and 95\% $\mathrm{CI}=-0.024 \sim 0.422$ ) for untrained group. While for the trained group, only BL attained significance level ( $p=0.045^{*}$ and $\left.95 \% \mathrm{CI}=-0.003 \sim 0.000\right)$.

\subsection{Cardiorespiratory Variables for Three Dumbbell Exercises (Exercise and Resting Period)}

During exercise, a significant difference was noted for MET $\left(p=0.000^{*}\right)$, RER $\left(p=0.000^{*} \sim 0.002^{*}\right)$, and HR $\left(p=0.000^{*} \sim 0.025^{*}\right)$ for all three exercises between the two groups, respectively, except RER for squat and HR for shoulder press. During the resting period, all of the parameters reached the level of significance $\left(p=0.000^{*} \sim 0.036^{*}\right)$ between two groups except RPE for shoulder press and deadlift while RER for squat (Table 5). 
Table 5. Cardiorespiratory variables for three dumbbell exercises (Exercise and resting period). $(\mathrm{n}=11)$.

\begin{tabular}{|c|c|c|c|c|c|c|c|c|}
\hline \multirow{2}{*}{$\begin{array}{l}\text { Exercise } \\
\text { Type }\end{array}$} & \multicolumn{4}{|c|}{ During Exercise } & \multicolumn{4}{|c|}{ During Rest } \\
\hline & Parameter & $\mathrm{UTr}(\mathrm{n}=5)$ & $\operatorname{Tr}(n=6)$ & $p$-Value & Parameter & $\mathrm{UTr}(\mathrm{n}=5)$ & $\operatorname{Tr}(n=6)$ & $p$-Value \\
\hline \multirow{7}{*}{$\begin{array}{l}\text { Shoulder } \\
\text { Press }\end{array}$} & MET & $1.30 \pm 0.29$ & $2.02 \pm 0.42$ & $0.000 *$ & MET & $1.36 \pm 0.24$ & $1.66 \pm 0.22$ & $0.000 *$ \\
\hline & RER & $1.15 \pm 0.15$ & $1.22 \pm 0.16$ & $0.002 *$ & RER & $1.06 \pm 0.13$ & $1.14 \pm 0.11$ & 0.000 * \\
\hline & \multirow[t]{5}{*}{ HR } & \multirow{5}{*}{$\begin{array}{c}109.39 \pm \\
10.50\end{array}$} & \multirow[t]{5}{*}{$\begin{array}{c}112.13 \pm \\
17.27\end{array}$} & \multirow[t]{5}{*}{0.190} & HR & $87.04 \pm 11.62$ & $95.07 \pm 16.84$ & $0.000 *$ \\
\hline & & & & & SBP & $110.60 \pm 5.08$ & $128.08 \pm 14.04$ & $0.000 *$ \\
\hline & & & & & DBP & $64.07 \pm 9.06$ & $73.86 \pm 7.38$ & $0.000 *$ \\
\hline & & & & & BL & $47.83 \pm 18.33$ & $60.00 \pm 23.60$ & 0.000 * \\
\hline & & & & & RPE & $10.40 \pm 1.51$ & $10.03 \pm 1.78$ & 0.118 \\
\hline \multirow{7}{*}{ Deadlift } & MET & $2.71 \pm 0.58$ & $3.13 \pm 0.73$ & $0.000 *$ & MET & $2.73 \pm 0.30$ & $3.12 \pm 0.39$ & 0.000 * \\
\hline & RER & $1.04 \pm 0.13$ & $1.11 \pm 0.15$ & $0.000 *$ & RER & $1.01 \pm 0.11$ & $1.06 \pm 0.17$ & $0.010 *$ \\
\hline & \multirow[t]{5}{*}{ HR } & \multirow{5}{*}{$\begin{array}{c}108.21 \pm \\
15.06\end{array}$} & \multirow{5}{*}{$\begin{array}{c}112.77 \pm \\
13.37\end{array}$} & \multirow[t]{5}{*}{$0.025^{*}$} & HR & $99.28 \pm 15.01$ & $106.62 \pm 18.36$ & $0.003 *$ \\
\hline & & & & & SBP & $125.00 \pm 5.59$ & $143.44 \pm 13.50$ & $0.000 *$ \\
\hline & & & & & DBP & $72.17 \pm 7.19$ & $80.22 \pm 6.51$ & 0.000 * \\
\hline & & & & & $\mathrm{BL}$ & $49.47 \pm 14.86$ & $67.92 \pm 28.91$ & 0.000 * \\
\hline & & & & & RPE & $10.60 \pm 1.90$ & $10.69 \pm 1.95$ & 0.732 \\
\hline \multirow{7}{*}{ Squat } & MET & $2.70 \pm 0.44$ & $3.42 \pm 0.77$ & $0.000 *$ & MET & $2.97 \pm 0.28$ & $3.51 \pm 0.41$ & 0.000 * \\
\hline & RER & $1.11 \pm 0.17$ & $1.16 \pm 0.16$ & 0.055 & RER & $1.18 \pm 0.11$ & $1.18 \pm 0.13$ & 0.962 \\
\hline & \multirow[t]{5}{*}{ HR } & \multirow[t]{5}{*}{$\begin{array}{c}110.97 \pm \\
15.00\end{array}$} & \multirow[t]{5}{*}{$\begin{array}{c}120.57 \pm \\
19.75\end{array}$} & \multirow[t]{5}{*}{$0.000 *$} & HR & $\begin{array}{c}107.49 \pm \\
15.48\end{array}$ & $115.34 \pm 22.17$ & $0.005 *$ \\
\hline & & & & & SBP & $122.50 \pm 7.98$ & $144.06 \pm 17.81$ & 0.000 * \\
\hline & & & & & DBP & $70.63 \pm 5.68$ & $78.06 \pm 6.63$ & $0.000 *$ \\
\hline & & & & & BL & $68.93 \pm 15.67$ & $78.75 \pm 30.16$ & $0.006^{*}$ \\
\hline & & & & & RPE & $11.07 \pm 2.12$ & $11.69 \pm 2.05$ & $0.036^{*}$ \\
\hline
\end{tabular}

One-way ANOVA: Mean \pm SD values. UTr, untrained; Tr, trained groups; * indicates a significant difference $p<0.050$; RER, respiratory exchange ratio; HR, heart rate; SBP, systolic blood pressure; DBP, diastolic blood pressure; BL, blood lactate; RPE, Borg rate of perceived exertion.

\section{Discussion}

This study aimed to predict GEE models for exercise and resting periods during three dumbbell exercises including shoulder press, deadlift, and squat for untrained and trained healthy participants. During exercise, RER, HR, SBP, and BL predicted significant models with MET for untrained and trained groups (Table 3). While RER, HR, SBP, DBP, and RPE reported significant models during the resting period for the untrained and $\mathrm{BL}$ for the trained groups (Table 4).

Most of the available literature found regression models to predict EE using body composition $(p<0.001)$, ambient temperature $(p=0.004)$ [10], and exercise intensity-loads and volume $\left(\mathrm{R}^{2}=0.61\right.$ and $\left.p<0.05\right)$ [9] etc. A study by Farinatti and Neto reported that total energy expenditure (EE) is mainly affected by muscle mass $(p>0.001)$ during five sets of ten repetitions with 15 RM loads of moderate-intensity horizontal leg press and chest fly with 1 and $3 \mathrm{~min}$ rest intervals [21]. Another study predicted regression models for bench press $\mathrm{Y}=0.132+(0.031)\left(\mathrm{X}_{1}\right)+(0.01)\left(\mathrm{X}_{2}\right), \mathrm{R}^{2}=0.728$ and parallel squat $\mathrm{Y}=-1.424+(0.022)$ $\left(X_{1}\right)+(0.035)\left(X_{2}\right), R^{2}=0.656$ with low intensity for consecutive 5 min exercise-where $\mathrm{Y}$ is $\mathrm{VO}_{2}, \mathrm{X}_{1}$ is the load measured in $\mathrm{kg}$ and $\mathrm{X}_{2}$ is the distance in $\mathrm{cm}$ [1]. One published study predicted EE during traditional strength training in 30 trained healthy participants using triaxial accelerometry found a correlation with the axis and location of accelerometer as vertical axis $(R=0.67)$, the horizontal axis $(R=0.43)$, third axis $(R=0.36)$, and the sum of three axes $(R=0.50)$ counts at the waist [22]. Not one study has predicted MET from cardiorespiratory variables during moderate-intensity interval resistance training. It is challenging to compare our predicted models due to the lack of research on the GEE modeling during resistance training exercises.

The primary goal of this study was to estimate GEE models for MET. These models were computed into two categories: (1) exercise and (2) resting models. During exercise, group models have low QIC (UTr $=74)$ for untrained group and predicted MET significantly with two parameters RER and HR (95\% CI $\left.=0.009 \sim 0.789, p=0.000^{*} \sim 0.004^{*}\right)$ while trained group has QIC ( $\mathrm{Tr}=187)$ and predicted MET with RER, HR, SBP, and BL $(95 \%$ 
$\left.\mathrm{CI}=-0.012 \sim 0.915, p=0.000^{*} \sim 0.033^{*}\right)$. It explains the difference in correlation between the two groups. As the trained group has more parameters' correlation which might be due to the previous experience of resistance training and higher loads for three exercises during RM testing (Table 3). Because the higher the lifted load, the greater the metabolic rate will be, so their cardiorespiratory parameters significantly differed from the untrained group (Table 5). Moreover, deadlift and squat exercises are more challenging due to many muscles involved in the lower body (quadriceps and hamstrings) and core (erector spinae and gluteals) than the shoulder press exercise which majorly involves upper body muscles (deltoid, serratus anterior, and latissimus dorsi, etc.). While during the resting period, only BL was significantly related with MET for the training group (QIC $=95,95 \%$ $\left.\mathrm{CI}=-0.003 \sim 0.000, p=0.045^{*}\right)$ but for the untrained group RER, HR, SBP, DBP, and RPE ( $\mathrm{QIC}=48,95 \% \mathrm{CI}=-0.024 \sim 0.422, p=0.000^{*} \sim 0.002^{*}$ ) significantly correlated with MET (Table 4). The results of this study observed that during exercise and rest periods, the untrained group has low QIC and more significant $p$-values than the training group models which are more predictive as per the criteria for evaluating these models $[18,20]$.

The cardiorespiratory variables included in this study were RER, HR, SBP, DBP, BL, and RPE (Table 5). During moderate-intensity interval RT, not all variables have been well-studied in the previous literature for healthy populations. The RER is an indirect measure of the relative utilization of carbohydrates and lipids to overall EE during a steady-state condition. Usually, a high RER reflects carbohydrate oxidation and a low RER indicates lipid oxidation [23]. Therefore, RER during exercise and resting period increased significantly for untrained and trained groups $\left(p=0.000^{*} \sim 0.002^{*}\right)$ for shoulder press and deadlift exercises. However, our study focused on RT protocol and did not include dietary assessment for participants, so the change in RER cannot be explained well.

Interval and RT elicit a greater rise in HR. Fluctuations are seen due to the interruptions in the continuity of the workout modalities [24]. During each repetition, HR responds similarly between eccentric and concentric phases [25]. Hunter et al. [26] reported an increase in heart rate during a traditional resistance training exercise and recovery period as $143 \pm 8.0$ and $119 \pm 12$ respectively. In the present study, HR values during exercise and resting period increased in both groups, but this increase was higher for the trained $(115.16 \pm 3.84$ and $105.68 \pm 8.30)$ than the untrained group $(109.52 \pm 1.13$ and $97.94 \pm 8.40)$ for all three exercises during six RT sessions. This increase could be explained by the higher workloads of the training group, which imposed more challenges on the cardiovascular system.

Based on the possible mechanisms for the change in cardiorespiratory variables during resistance training, blood pressure (BP) increases proportionally to the training load. The main reasons for the change in blood pressure are the use of the Valsalva maneuver, muscle mass activation, muscle action, and the intensity/duration of the set [25]. One study reported mean BPs of 320/250 and 345/245 mmHg during high-intensity leg press and squat in trained bodybuilders [27]. During the Valsalva maneuver, breath-holding increases intrathoracic and intra-abdominal pressure that leads to the increase in blood pressure proportionally $[28,29]$. Resting blood pressure may not be affected or reduced by RT. Strength-trained athletes have resting blood pressure that is average or below average [25]. According to a meta-analysis of the RT literature, SBP and DBP may be decreased by $2-4 \%[30,31]$. In our study, $\mathrm{BP}$ was recorded only during resting periods (2-5 min after exercise) as SBP (119.37 \pm 6.28 and $138.53 \pm 7.39)$ and DBP (68.96 \pm 3.51 and $77.38 \pm 2.64$ ) for untrained and training groups, respectively, which followed the same trend from previous studies but a slight increase in SBP was noted for the training group.

The BL can describe the anaerobic component of the RT. It was measured before the start of exercise and $5 \mathrm{~min}$ after each exercise for every training session. It changed for all three dumbbell exercises significantly $\left(p=0.000^{*} \sim 0.006^{*}\right)$ in both groups. 


\section{Limitations and Practical Applications}

(1) The sample size of this study was too small $(n=11)$ so the results cannot be generalized to the large healthy population. In the future, more research with a large sample size will be required to test this relationship including other upper and lower limb RT exercises with different speeds, RM loading, or including training sessions to failure. (2) The energy intake was not monitored in this study, which could also contribute and affect the relationship of MET with cardiorespiratory variables such as RER, HR, SBP, DBP, and BL, etc. An appropriate dietary assessment will be added for a well-controlled exercise study design. (3) The resistance training experience for the training group in this study was only 2 months and should be around 6 months to 1 year for a clearer comparison between healthy control and athletic population to translate study outcomes into the human population. (4) This is an initial pilot study to test the relationship between MET and cardiorespiratory variables through GEE modeling, however, in the future, this protocol will require testing on a large population to confirm this study's findings.

This is an important area of research to determine the highly related cardiorespiratory variables in designing reliable and accurate exercise protocols for the health and fitness purposes of healthy, obese, and clinical populations.

\section{Conclusions}

Based on the study's findings, the cardiorespiratory variables are significantly related to MET. During exercise, RER and HR significantly predicted MET for both groups along with additional parameters of SBP and BL for the training group. While during the resting period, RER, HR, SBP, DBP, and RPE related significantly for untrained and BL for the training group, respectively. The cardiorespiratory variables significantly changed for both groups.

Author Contributions: Conceptualization, M.A. and C.-W.P.; methodology, M.A., C.-H.L. and C.W.P.; software, M.A., C.-W.W. and C.-W.P.; validation, M.A., C.-H.L., J.-H.K. and C.-W.P.; formal analysis, M.A., M.-J.H. and C.-W.P.; investigation, M.A., M.-J.H. and C.-T.F.; resources, C.-H.L., J.-H.K., H.-C.C. and C.-W.P.; data curation, M.A., C.-W.W., B.-S.L. and C.-W.P.; writing-original draft preparation, M.A.; writing-review and editing, M.A., C.-H.L. and C.-W.P.; visualization, J.-H.K., J.-C.L. and H.-C.C.; supervision, C.-H.L., C.-W.W., J.-H.K., J.-C.L., H.-C.C., B.-S.L. and C.-W.P.; project administration, C.-H.L., C.-T.F. and J.-H.K.; funding acquisition, C.-W.P. All authors have read and agreed to the published version of the manuscript.

Funding: The present study was generously funded by the Ministry of Science and Technology (110-2314-B-038-001, 110-2314-B-305-001, 110-2811-E-038-500-MY3, 109-2221-E-038-005-MY3, and 109-2314-B-038-132) and the Higher Education Sprout Project by the Ministry of Education (DP2-10821121-01-N-08-02 and DP2-110-21121-01-N-02-01) of Taiwan.

Institutional Review Board Statement: The study was conducted according to guidelines of the Declaration of Helsinki, and approved by the Institutional Review Board (or Ethics Committee) of the TMU-Joint Institutional Review Board (IRB no: N202004023, 2 June 2020). The trial was registered at ClinicalTrials.gov (accessed on 11 September 2021) (NCT04532905).

Informed Consent Statement: Written informed consent was obtained from all participants involved in the study.

Data Availability Statement: The data presented in this study are all available in the article.

Acknowledgments: We would like to acknowledge our very dedicated research assistants, clinical staff at Taipei Medical University Hospital, and Shuang Ho Hospital for their untiring efforts in collecting data and recruiting participants in Taiwan and consultation from the department of statistics at Taipei Medical University for analyzing data.

Conflicts of Interest: The authors declare no conflict of interest. 


\begin{abstract}
Abbreviations
RT: resistance training; ACSM, American College of Sports Medicine; AHA, American Heart Association; EE, energy expenditure; IC, indirect calorimetry; MET, metabolic equivalent; $\mathrm{kg} / \mathrm{h}$; kilogram per hour; REE, resting energy expenditure; FFM, fat free mass; FM, fat mass; RM, repetition maximum; RER, respiratory exchange ratio; HR, heart rate; BP, blood pressure; SBP, systolic blood pressure; DBP, diastolic blood pressure; BL, blood lactate; RPE, Borg rate of perceived exertion; GEE, generalized estimating equations; IRB, institutional review board; PAR-Q, Physical Activity Readiness Questionnaire; $\mathrm{H}$, hour; $\mathrm{BMI}$, body-mass index; RPM, revolutions per minute; $\mathrm{S}$, second; Min, minute; $\mathrm{VO}_{2}$, oxygen consumption; $\mathrm{ml} / \mathrm{kg} / \mathrm{min}$, milliliter per kilogram per minute; $\mathrm{BPM}$, beats per minute; $\mathrm{mmHg}$, millimeter of mercury; mg/dl, milligram per deciliter; SPSS, Statistical Package for the Social Sciences; $B$, estimate; SE, standard error; CI, confidence interval; QIC, quasi-likelihood under independence model criterion; SD, standard deviation; ANOVA, analysis of variance; $n$, number of participant; $\mathrm{cm}$, centimeter; $\mathrm{kg} / \mathrm{m}^{2}$, kilogram per meter square; $\mathrm{kg}$, kilogram; UTr, untrained; Tr, trained.
\end{abstract}

\title{
References
}

1. Robergs, R.A.; Gordon, T.; Reynolds, J.; Walker, T.B. Energy expenditure during bench press and squat exercises. J. Strength Cond. Res. 2007, 21, 123-130. [CrossRef] [PubMed]

2. Pollock, M.L.; Gaesser, G.A.; Butcher, J.D.; Després, J.P.; Dishman, R.K.; Franklin, B.A.; Garber, C. ACSM Position Stand: The Recommended Quantity and Quality of Exercise for Developing and Maintaining Cardiorespiratory and Muscular Fitness, and Flexibility in Healthy Adults. Med. Sci. Sports Exerc. 1998, 30, 975-991. [CrossRef]

3. Scala, D.; McMillan, J.; Blessing, D.; Rozenek, R.; Stone, M. Metabolic Cost of a Preparatory Phase of Training in Weight Lifting: A Practical Observation. J. Strength Cond. Res. 1987, 1, 48-52. [CrossRef]

4. De Mello Meirelles, C.; Gomes, P. Acute effects of resistance exercise on energy expenditure: Revisiting the impact of the training variables. Rev. Bras. Med. Esporte 2004, 10, 131-138.

5. Wilmore, J.H.; Parr, R.B.; Ward, P.; A Vodak, P.; Barstow, T.J.; Pipes, T.V.; Grimditch, G.; Leslie, P. Energy cost of circuit weight training. Med. Sci. Sports 1978, 10, 75-78. [PubMed]

6. Achamrah, N.; Delsoglio, M.; De Waele, E.; Berger, M.M.; Pichard, C. Indirect calorimetry: The 6 main issues. Clin. Nutr. 2021, 40, 4-14. [CrossRef] [PubMed]

7. $\quad$ Ainsworth, B.E.; Haskell, W.L.; Whitt, M.C.; Irwin, M.L.; Swartz, A.M.; Strath, S.J.; O’Brien, W.L.; Bassett, D.R., Jr.; Schmitz, K.H.; Emplaincourt, P.O.; et al. Compendium of Physical Activities: An update of activity codes and MET intensities. Med. Sci. Sports Exerc. 2000, 32, 498-516. [CrossRef]

8. Ainsworth, B.E.; Haskell, W.L.; Herrmann, S.D.; Meckes, N.; Bassett, D.R.; Tudor-Locke, C. Compendium of Physical Activities: A second update of codes and MET values. Med. Sci. Sports Exerc. 2011, 43, 1575-1581. [CrossRef]

9. João, G.A.; Rodriguez, D.; Tavares, L.D.; Rica, R.L.; Júnior, N.C.; Reis, V.M.; Junior, F.L.P.; Baker, J.S.; Bocalini, D.S.; Júnior, A.F. Energy expenditure estimation of a moderate-intensity strength training session. Cogent Med. 2020, 7, 1794500. [CrossRef]

10. Frings-Meuthen, P.; Henkel, S.; Boschmann, M.; Chilibeck, P.D.; Cruz, J.R.A.; Hoffmann, F.; Möstl, S.; Mittag, U.; Mulder, E.; Rittweger, N. Resting Energy Expenditure of Master Athletes: Accuracy of Predictive Equations and Primary Determinants. Front. Physiol. 2021, 12, 278. [CrossRef]

11. Warburton, D.E.; Jamnik, V.; Bredin, S.S.; Shephard, R.J.; Gledhill, N. The 2019 physical activity readiness questionnaire for everyone (PAR-Q+) and electronic physical activity readiness medical examination (ePARmed-X+). Health Fit. J. Canada. 2018, 11, 80-83.

12. Kubo, Y.; Fujita, D.; Sugiyama, S.; Hosokawa, M.; Nishida, Y. Pulmonary oxygen uptake on-kinetics can predict acute physiological responses to resistance exercise training in healthy young men. Clin. Physiol. Funct. Imaging 2019, 39, 339-344. [CrossRef]

13. Davis, C. Upright Bicycle and Treadmill Stress Echocardiography Techniques and Technical Hints for the Sonographer. J. Am. Soc. Echocardiogr. 1994, 7, 194-200. [CrossRef]

14. Poole, D.C.; Wilkerson, D.P.; Jones, A.M. Validity of criteria for establishing maximal O2 uptake during ramp exercise tests. Graefe's Arch. Clin. Exp. Ophthalmol. 2008, 102, 403-410. [CrossRef]

15. Shimano, T.; Kraemer, W.J.; Spiering, B.A.; Volek, J.S.; Hatfield, D.L.; Silvestre, R.; Vingren, J.L.; Fragala, M.S.; Maresh, C.M.; Fleck, S.J.; et al. Relationship Between the Number of Repetitions and Selected Percentages of One Repetition Maximum in Free Weight Exercises in Trained and Untrained Men. J. Strength Cond. Res. 2006, 20, 819-823. [CrossRef] [PubMed]

16. Lyristakis, P.; Ball, N.; McKune, A.J. Reliability of methods to measure energy expenditure during and after resistance exercise. Appl. Physiol. Nutr. Metab. 2019, 44, 1276-1282. [CrossRef] [PubMed]

17. Burton, P.; Gurrin, L.; Sly, P. Extending the simple linear regression model to account for correlated responses: An introduction to generalized estimating equations and multi-level mixed modelling. Stat. Med. 1998, 17, 1261-1291. [CrossRef]

18. Piggott, B.; Müller, S.; Chivers, P.; Cripps, A.; Hoyne, G. Interdisciplinary Sport Research Can Better Predict Competition Performance, Identify Individual Differences, and Quantify Task Representation. Front. Sports Act. Living 2020, 2, 14. [CrossRef] 
19. Tan, S.H.; Tan, S.B. The correct interpretation of confidence intervals. Proc. Singap. Healthc. 2010, 19, 276-278. [CrossRef]

20. Pan, W. Akaike's information criterion in generalized estimating equations. Biometrics 2001, 57, 120-125. [CrossRef]

21. Farinatti, P.T.; Neto, A.G.C. The effect of Between-Set Rest Intervals on the Oxygen Uptake During and After Resistance Exercise Sessions Performed with Large- and Small-Muscle Mass. J. Strength Cond. Res. 2011, 25, 3181-3190. [CrossRef]

22. Stec, M.J.; Rawson, E.S. Estimation of Resistance Exercise Energy Expenditure Using Triaxial Accelerometry. J. Strength Cond. Res. 2012, 26, 1413-1422. [CrossRef]

23. Ramos-Jiménez, A.; Hernández-Torres, R.P.; Torres-Durán, P.V.; Romero-Gonzalez, J.; Mascher, D.; Posadas-Romero, C.; JuárezOropeza, M.A. The Respiratory Exchange Ratio is Associated with Fitness Indicators Both in Trained and Untrained Men: A Possible Application for People with Reduced Exercise Tolerance. Clin. Med. Circ. Respir. Pulm. Med. 2008, 2, 1-9. [CrossRef]

24. Ratamess, N.A.; Kraemer, W.J.; Volek, J.S.; Maresh, C.M.; VanHeest, J.L.; Sharman, M.; Rubin, M.R.; French, D.N.; Vescovi, J.D.; Silvestre, R.; et al. Androgen receptor content following heavy resistance exercise in men. J. Steroid Biochem. Mol. Biol. 2005, 93, 35-42. [CrossRef] [PubMed]

25. Fleck, S.J. Cardiovascular Responses to Strength Training. Strength Power Sport 2003, 2, 387-406. [CrossRef]

26. Hunter, G.R.; Seelhorst, D.; Snyder, S. Comparison of metabolic and heart rate responses to super slow vs. traditional resistance training. J. Strength. Cond. Res. 2003, 17, 76-81. [PubMed]

27. MacDougall, J.D.; Tuxen, D.; Sale, D.G.; Moroz, J.R.; Sutton, J.R. Arterial blood pressure response to heavy resistance exercise. J. Appl. Physiol. 1985, 58, 785-790. [CrossRef]

28. Haykowsky, M.J.; Dressendorfer, R.; Taylor, D.; Mandic, S.; Humen, D. Resistance Training and Cardiac Hypertrophy. Sports Med. 2002, 32, 837-849. [CrossRef] [PubMed]

29. Palatini, P.; Mos, L.; Munari, L.; Valle, F.; Del Torre, M.; Rossi, A.; Varotto, L.; Macor, F.; Martina, S.; Pessina, A.C.; et al. Blood pressure changes during heavy-resistance exercise. J. Hypertens. 1989, 7, 72-73. [CrossRef]

30. Kelley, G.A.; Kelley, K.S. Progressive resistance exercise and resting blood pressure: A meta-analysis of randomized controlled trials. Hypertension 2000, 35, 838-843. [CrossRef]

31. Kelley, G. Dynamic resistance exercise and resting blood pressure in adults: A meta-analysis. J. Appl. Physiol. 1997, 82, 1559-1565. [CrossRef] [PubMed] 